\title{
ON POWER SUMS OF MATRICES OVER A FINITE COMMUTATIVE RING
}

\author{
P. FORTUNY, J.M. GRAU, A.M. OLLER-MARCÉN, AND I.F. RÚA
}

\begin{abstract}
In this paper we deal with the problem of computing the sum of the $k$-th powers of all the elements of the matrix $\operatorname{ring} \mathbb{M}_{d}(R)$ with $d>1$ and $R$ a finite commutative ring. We completely solve the problem in the case $R=\mathbb{Z} / n \mathbb{Z}$ and give some results that compute the value of this sum if $R$ is an arbitrary finite commutative ring $R$ for many values of $k$ and $d$. Finally, based on computational evidence and using some technical results proved in the paper we conjecture that the sum of the $k$-th powers of all the elements of the matrix ring $\mathbb{M}_{d}(R)$ is always 0 unless $d=2, \operatorname{card}(R) \equiv 2(\bmod 4)$, $1<k \equiv-1,0,1(\bmod 6)$ and the only element $e \in R \backslash\{0\}$ such that $2 e=0$ is idempotent, in which case the $\operatorname{sum}$ is $\operatorname{diag}(e, e)$.
\end{abstract}

\section{INTRODUCTION}

For a ring $R$ we denote by $\mathbb{M}_{d}(R)$ the ring of $d \times d$ matrices over $R$. Now, given an integer $k \geq 1$ we define the sum

$$
S_{k}^{d}(R):=\sum_{M \in \mathbb{M}_{d}(R)} M^{k} .
$$

This paper deals with the computation of $S_{k}^{d}(R)$ in the case when $R$ is finite and commutative.

When $d=1$, the problem of computing $S_{k}^{1}(R)$ is completely solved only for some particular families of finite commutative rings. If $R$ is a finite field $\mathbb{F}_{q}$, the value of $S_{k}^{1}\left(\mathbb{F}_{q}\right)$ is well-known. If $R=\mathbb{Z} / n \mathbb{Z}$ the study of $S_{k}^{1}(\mathbb{Z} / n \mathbb{Z})$ dates back to 1840 ] and has been completed in various works $[2,5$, 7]. Finally, the case $R=\mathbb{Z} / n \mathbb{Z}[i]$ has been recently solved in [3. For those rings, we have the following result.

Theorem 1. Let $k \geq 1$ be an integer.

i) Finite fields:

$$
S_{k}^{1}\left(\mathbb{F}_{q}\right)= \begin{cases}-1, & \text { if }(q-1) \mid k \\ 0, & \text { otherwise. }\end{cases}
$$

ii) Integers modulo $n$ :

$$
S_{k}^{1}(\mathbb{Z} / n \mathbb{Z})= \begin{cases}-\sum_{p|n, p-1| k} \frac{n}{p}, & \text { if } k \text { is even or } k=1 \text { or } n \not \equiv 0 \quad(\bmod 4) ; \\ 0, & \text { otherwise. }\end{cases}
$$


iii) Gaussian integers modulo $n$ :

$$
S_{k}^{1}(\mathbb{Z} / n \mathbb{Z}[i])= \begin{cases}\frac{n}{2}(1+i), & \text { if } k>1 \text { is odd and } n \equiv 2 \quad(\bmod 4) \\ -\sum_{p \in \mathcal{P}(k, n)} \frac{n^{2}}{p^{2}}, & \text { otherwise. }\end{cases}
$$

where

$$
\mathcal{P}(k, n):=\left\{\text { prime } p: p \| n, p^{2}-1 \mid k, p \equiv 3 \quad(\bmod 4)\right\}
$$

and $p \| n$ means that $p \mid n$, but $p^{2} \nmid n$.

On the other hand, if $d>1$ the problem has been only solved when $R$ is a finite field [1]. In particular, the following result holds.

Theorem 2. Let $k, d \geq 1$ be integers. Then $S_{k}^{d}\left(\mathbb{F}_{q}\right)=0$ unless $q=2=d$ and $1<k \equiv-1,0,1(\bmod 6)$ in which case $S_{k}^{d}\left(\mathbb{F}_{q}\right)=I_{2}$.

In this paper we deal with the computation of $S_{k}^{d}(R)$ with $d>1$ and $R$ a finite commutative ring. In particular Section 2 is devoted to completely determine the value of $S_{k}^{d}(R)$ in the case $R=\mathbb{Z} / n \mathbb{Z}$ (that we usually write as $\mathbb{Z}_{n}$ ). In Section 3 we give some technical results regarding sums of non-commutative monomials over $\mathbb{Z} / n \mathbb{Z}$ which will be used in Section 4 to compute $S_{k}^{d}(R)$ for an arbitrary finite commutative ring $R$ in many cases. Finally, we close the paper in Section 5 with the following conjecture based on strong computational evidence

Conjecture 1. Let $d>1$ and let $R$ be a finite commutative ring. Then $S_{k}^{d}(R)=0$ unless the following conditions hold:

(1) $d=2$,

(2) $\operatorname{card}(R) \equiv 2(\bmod 4)$ and $1<k \equiv-1,0,1(\bmod 6)$,

(3) The unique element $e \in R \backslash\{0\}$ such that $2 e=0$ is idempotent.

Moreover, in this case

$$
S_{k}^{d}(R)=\left(\begin{array}{ll}
e & 0 \\
0 & e
\end{array}\right)
$$

\section{Power Sums of MATRICES OVER $\mathbb{Z}_{n}$}

In what follows we will consider integers $n, d>1$. For the sake of simplicity, $M_{n}^{d}$ will denote the set of integer matrices with entries in the range $\{0, \ldots, n-1\}$. Furthermore, for an integer $k \geq 1$, let $S_{k}^{d}(n)=\sum_{M \in M_{n}^{d}} M^{k}$. Our main goal in this section will be to compute the value of $S_{k}^{d}(n)$ modulo $n$. This is exactly the sum $S_{k}^{d}(\mathbb{Z} / n \mathbb{Z})$.

We start with the prime case. If $n=p$ is a prime, we have the following result 1. Corollary 3.2]

Proposition 1. Let $p$ be a prime. Then, $S_{k}^{d}(p) \equiv 0(\bmod p)$ unless $d=p=2$.

Thus, the case $n=2$ must be studied separately. In fact, we have

Proposition 2.

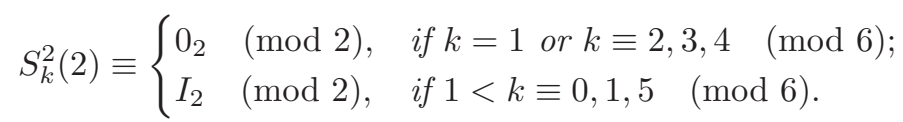


Proof. For every $M \in M_{n}^{2}$ it holds that $M^{2} \equiv M^{8}(\bmod 2)$. As a consequence $S_{k}^{2}(2) \equiv S_{k+6}^{2}(2)(\bmod 2)$ for every $k>1$. Thus, the result follows just computing $S_{k}^{2}(2)$ for $1 \leq k \leq 7$.

Now, we turn to the prime power case. The following lemma is straightforward

Lemma 1. Let $p$ be a prime. Then, any element $M$ in $M_{p^{s+1}}^{d}$ can be uniquely written in the form $A+p^{s} B$, where $A \in M_{p^{s}}^{d}, B \in M_{p}^{d}$.

Using this lemma we can prove the following useful result.

Proposition 3. Let $p$ be a prime. Then, $S_{k}^{d}\left(p^{s+1}\right) \equiv p^{d^{2}} S_{k}^{d}\left(p^{s}\right)\left(\bmod p^{s+1}\right)$.

Proof. By the previous lemma we have

$$
S_{k}^{d}\left(p^{s+1}\right)=\sum_{M \in M_{p^{s}}^{d}} M^{k}=\sum_{A \in M_{p^{s}}^{d}} \sum_{B \in M_{p}^{d}}\left(A+p^{s} B\right)^{k} .
$$

Using the non-commutative version of the binomial theorem we have that

$$
\left(A+p^{s} B\right)^{k} \equiv A^{k}+p^{s} \sum_{t=1}^{k} A^{k-t} B A^{t-1} \quad\left(\bmod p^{s+1}\right) .
$$

Thus, combining this with (10) we obtain

$$
\begin{aligned}
S_{k}^{d}\left(p^{s+1}\right) & \equiv \sum_{B \in M_{p}^{d}}\left(\sum_{A \in M_{p^{s}}^{d}} A^{k}\right)+\sum_{t=1}^{k} \sum_{A \in M_{p^{s}}^{d}} A^{k-t}\left(p^{s} \sum_{B \in M_{p}^{d}} B\right) A^{t-1} \\
& \equiv p^{d^{2}} S_{k}^{d}\left(p^{s}\right)+\sum_{t=1}^{k} \sum_{A \in M_{p^{s}}^{d}} A^{k-t}\left(p^{s} S_{1}^{d}(p)\right) A^{t-1} \\
& \equiv p^{d^{2}} S_{k}^{d}\left(p^{s}\right) \quad\left(\bmod p^{s+1}\right)
\end{aligned}
$$

because $S_{1}^{d}(p) \equiv 0(\bmod p)$ by Propositions 1 and 2 (depending on whether $p$ is odd or not).

Remark. Note that Proposition 3 implies that if $S_{k}^{d}\left(p^{s}\right) \equiv 0\left(\bmod p^{s}\right)$, then also $S_{k}^{d}\left(p^{s+1}\right) \equiv 0\left(\bmod p^{s+1}\right)$.

As a consequence we get the following result which extends Proposition 1.

Corollary 1. $S_{k}^{d}\left(p^{s}\right) \equiv 0\left(\bmod p^{s}\right)$ unless $d=p=2$ and $s=1$.

Proof. If $p=d=2$, then Proposition 1 implies that $S_{k}^{2}(4) \equiv 2{ }^{4} S_{k}^{2}(2) \equiv 0(\bmod 4)$, so the previous remark leads to $S_{k}^{2}\left(2^{s}\right) \equiv 0\left(\bmod 2^{s}\right)$, for every $s>1$. On the other hand, if $d$ or $p$ is odd, then we know by Proposition 1 that $S_{k}^{d}(p) \equiv 0(\bmod p)$. Again, the remark gives us $S_{k}^{d}\left(p^{s}\right) \equiv 0$, by induction for all $s \geq 1$.

In order to study the general case the following lemma will be useful. It is an analogue of [6, Lemma 3 i)]

Lemma 2. If $m \mid n$, then $S_{k}^{d}(n) \equiv\left(\frac{n}{m}\right)^{d^{2}} S_{k}^{d}(m)(\bmod m)$. 
Proof. Given a matrix $M \in M_{n}^{d}$, let $M=\left(m_{i, j}\right)$ with $1 \leq i, j \leq d$. Then,

$$
\begin{aligned}
S_{k}^{d}(n) & =\sum_{M \in M_{n}^{d}} M^{k}=\sum_{0 \leq m_{i, j} \leq n-1}\left(m_{i, j}\right)^{k} \\
& \equiv\left(\frac{n}{m}\right)^{d^{2}} \sum_{0 \leq m_{i, j} \leq m-1}\left(m_{i, j}\right)^{k}=S_{k}^{d}(m) \quad(\bmod m)
\end{aligned}
$$

Now, we can prove the main result of this section.

Theorem 3. The following congruence modulo $n$ holds:

$$
S_{k}^{d}(n) \equiv \begin{cases}\frac{n}{2} \cdot I_{2}, & \text { if } d=2, n \equiv 2 \quad(\bmod 4) \text { and } 1<k \equiv 0,1,5 \quad(\bmod 6) ; \\ 0_{2}, & \text { otherwise. }\end{cases}
$$

Proof. Let $n=2^{s} p_{1}^{r_{1}} \cdots p_{t}^{r_{t}}$ be the prime power decomposition of $n$.

If $1 \leq i \leq t$, we have by Lemma 2 and Corollary 1 that

$$
S_{k}^{d}(n) \equiv\left(\frac{n}{p_{i}^{r_{i}}}\right)^{d^{2}} S_{k}^{d}\left(p_{i}^{r_{i}}\right) \equiv 0 \quad\left(\bmod p_{i}^{r_{i}}\right) .
$$

On the other hand, using again Lemma 2 we have that

$$
S_{k}^{d}(n) \equiv\left(\frac{n}{2^{s}}\right)^{d^{2}} S_{k}^{d}\left(2^{s}\right) \quad\left(\bmod 2^{s}\right) .
$$

Hence, Corollary 1 implies that $S_{k}^{d}(n) \equiv 0\left(\bmod 2^{s}\right)$ unless $d=p=2$ and $s=1$.

To conclude, it is enough to apply Proposition 2 together with the Chinese Remainder Theorem.

The following corollary easily follows from Theorem 3 and it confirms the conjecture stated in the sequence A017593 from the OEIS 8 .

Corollary 2. $S_{n}^{2}(n) \not \equiv 0(\bmod n)$ if and only if $n \equiv 6(\bmod 12)$.

As a further application of Theorem 3 application we are going to compute the sum of the powers of the Hamilton quaternions over $\mathbb{Z} / n \mathbb{Z}$.

Proposition 4. For every $n \in \mathbb{N}$ and $l>0$, it holds that

$$
\sum_{z \in \mathbb{Z}_{n}[i, j, k]} z^{l}=0
$$

Proof. Since for all $z \in \mathbb{Z}_{2}[i, j, k]$ we have that $z^{2} \in \mathbb{Z}_{2}$, we deduce that $z^{4}=z^{2}$, and so it can be straightforwardly checked that

$$
\sum_{z \in \mathbb{Z}_{2}[i, j, k]} z^{l}=0
$$

Now, if $s>1$, observing that

$$
\mathbb{Z}_{2^{s}}[i, j, k] \cong\left\{\left(\begin{array}{cccc}
a & b & c & d \\
-b & a & -d & c \\
-c & d & a & -b \\
-d & -c & b & a
\end{array}\right): a, b, c, d \in \mathbb{Z}_{2^{s}}\right\}
$$


we can adapt Lemma 11. Proposition 3 and Corollary 1 to inductively obtain that

$$
\sum_{z \in \mathbb{Z}_{2 s}[i, j, k]} z^{l}=0 .
$$

Finally, if $n=2^{s} m$ with $m$ odd we know [4, Theorem 4] that

$$
\mathbb{Z}_{n}[i, j, k] \cong \mathbb{Z}_{2^{s}}[i, j, k] \times \mathbb{Z}_{m}[i, j, k] \cong \mathbb{Z}_{2^{s}}[i, j, k] \times \mathbb{M}_{2}\left(\mathbb{Z}_{m}\right)
$$

and the result follows from Theorem 3

\section{Sums of NON-COMMUTATiVE MONOMials OVER $\mathbb{Z}_{n}$}

We will now consider a more general setting. Let $r \geq 1$ be an integer and consider $w\left(x_{1}, \ldots, x_{r}\right)$ a monomial in the non-commuting variables $\left\{x_{1}, \ldots, x_{r}\right\}$ of total degree $k$. In this situation, we define the sum

$$
S_{w}^{d}(n):=\sum_{A_{1}, \ldots, A_{r} \in M_{n}^{d}} w\left(A_{1}, \ldots, A_{r}\right) .
$$

Note that if $r=1$, then $w\left(x_{1}\right)=x_{1}^{k}$ and $S_{w}^{d}(n)=S_{k}^{d}(n)$ so we recover the situation from Section 2, Thus, in what follows we assume $r>1$.

We want to study the value of $S_{w}^{d}(n)$ modulo $n$. To do so we first introduce two technical lemmas that extend [1, Lemma 2.3].

Lemma 3. Let $\tau \geq 1$ be an integer and let $\beta_{i}>0$ for every $1 \leq i \leq \tau$. If $p$ is an odd prime,

$$
\sum_{x_{1}, \ldots, x_{\tau}} x_{1}^{\beta_{1}} \cdots x_{\tau}^{\beta_{\tau}} \equiv \begin{cases}\left(-p^{s-1}\right)^{\tau}, & \text { if } p-1 \mid \beta_{i} \text { for every } i ; \quad\left(\bmod p^{s}\right) \\ 0, & \text { otherwise. }\end{cases}
$$

where the sum is extended over $x_{1}, \ldots, x_{\tau}$ in the range $\left\{0, \ldots, p^{s}-1\right\}$. Also, if some $\beta_{i}=0$, then $\sum_{x_{1}, \ldots, x_{\tau}} x_{1}^{\beta_{1}} \cdots x_{\tau}^{\beta_{\tau}} \equiv 0\left(\bmod p^{s}\right)$.

Proof. It is enough to apply [6. Lemma 3 ii)] which states that

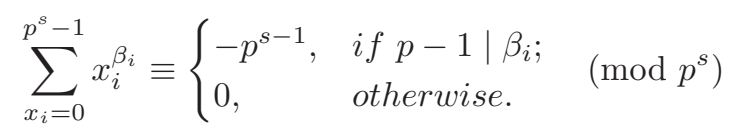

for every $1 \leq i \leq \tau$. Observe that, if $\beta_{i}=0$, then:

$$
\sum_{x_{1}, \ldots, x_{\tau}} x_{1}^{\beta_{1}} \cdots x_{\tau}^{\beta_{\tau}}=\sum_{x_{i}} \sum_{x_{j}, j \neq i} x_{1}^{\beta_{1}} \cdots x_{i-1}^{\beta_{i-1}} x_{i+1}^{\beta_{i+1}} \cdots x_{\tau}^{\beta_{\tau}} \equiv 0 \quad\left(\bmod p^{s}\right)
$$

Remark. Observe that in the previous situation, if $\tau \geq 2$ and $s>1$, it easily follows that $\sum_{x_{1}, \ldots, x_{\tau}} x_{1}^{\beta_{1}} \cdots x_{\tau}^{\beta_{\tau}} \equiv 0\left(\bmod p^{s}\right)$ regardless the values of $\beta_{i} \geq 0$.

Lemma 4. Let $\tau \geq 1$ be an integer and let $\beta_{i}>0$ for every $1 \leq i \leq \tau$. Then,

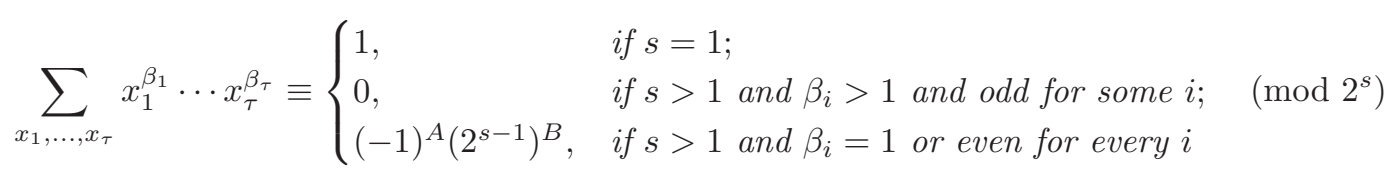


where the sum is extended over $x_{1}, \ldots, x_{\tau}$ in the range $\left\{0, \ldots, 2^{s}-1\right\}, A=\operatorname{card}\left\{\beta_{i}\right.$ :

$\left.\beta_{i}=1\right\}$ and $B=\operatorname{card}\left\{\beta_{i}: \beta_{i}\right.$ is even $\}$. Also, if some $\beta_{i}=0$, then $\sum_{x_{1}, \ldots, x_{\tau}} x_{1}^{\beta_{1}} \cdots x_{\tau}^{\beta_{\tau}} \equiv$ $0\left(\bmod 2^{s}\right)$.

Proof. It is enough to apply [6, Lemma 3 iii)] which states that

$$
\sum_{x_{i}=0}^{2^{s}-1} x_{i}^{\beta_{i}} \equiv\left\{\begin{array}{ll}
2^{s-1}, & \text { if } s=1 \text { or } s>1 \text { and } \beta_{1}>1 \text { is even; } \\
-1, & \text { if } s>1 \text { and } \beta_{i}=1 ; \\
0, & \text { if } s>1 \text { and } \beta_{1}>1 \text { is odd. }
\end{array} \quad\left(\bmod p^{s}\right)\right.
$$

for every $1 \leq i \leq \tau$. The proof of the case when some $\beta_{i}=0$ is identical to that of the previous lemma.

As a consequence, we get the following results.

Proposition 5. Let $p$ be an odd prime and let $s>1$ be an integer. Then,

$$
S_{w}^{d}\left(p^{s}\right) \equiv 0 \quad\left(\bmod p^{s}\right) .
$$

Proof. Let $A_{l}=\left(a_{i, j}^{l}\right)_{1 \leq i, j \leq d}$ for every $1 \leq l \leq r$. Note that each entry in the matrix $S_{w}^{d}\left(p^{s}\right)$ is a homogeneous polynomial in the variables $a_{i, j}^{l}$. Observe also that these variables are summation indexes in the range $\left\{0, \ldots, p^{s}-1\right\}$. Hence, the number of variables is $r d^{2}>2$ and, since $s>1$, the Remark 3 can be applied to the sum of its monomials, and the result follows.

Proposition 6. Let $s>1$ be an integer. Assume that one of the following conditions holds:

i) $k \leq r d^{2}$,

ii) $k>r d^{2}$ and $k+r d^{2}$ is even.

Then, $S_{w}^{d}\left(2^{s}\right) \equiv 0\left(\bmod 2^{s}\right)$.

Proof. Just like in the previous proposition each entry in the matrix $S_{w}^{d}\left(2^{s}\right)$ is a homogeneous polynomial in the $r d^{2}$ variables $a_{i, j}^{l}$. Hence, it is a sum of elements of the form

$$
\sum_{a_{i, j}^{l} \in \mathbb{Z}_{2 s}} \prod\left(a_{i, j}^{l}\right)^{\beta_{i, j, l}} .
$$

Observe that $\sum_{i, j, l} \beta_{i, j, l}=k$ so, if $k<r d^{2}$ it follows that some $\beta_{i, j, l}=0$, and so each monomial sum is $0 \bmod 2^{s}$ (because of Lemma 31). Therefore, each entry in the matrix $S_{w}^{d}(p)$ is $0\left(\bmod 2^{s}\right)$ in this case, as claimed.

Now, assume that $k \geq r d^{2}$ and $k+r d^{2}$ is even (in particular if $k=r d^{2}$ ). Due to Lemma 4 an element $\sum_{a_{i, j}^{l} \in \mathbb{Z}_{2^{s}}} \prod\left(a_{i, j}^{l}\right)^{\beta_{i, j, l}}$ is $0\left(\bmod 2^{s}\right)$ unless in one of its monomials the set of $r d^{2}$ exponents $\beta_{i, j, l}$ is formed by exactly $r d^{2}-1$ ones and 1 even value. But in this case $k=\left(r d^{2}-1\right)+2 \alpha$ so $k+r d^{2}$ is odd, a contradiction. Consequently, each entry in the matrix $S_{w}^{d}(p)$ is also $0\left(\bmod 2^{s}\right)$ in this case and the result follows.

As Remark 3 and Lemma 4 point out, the case $s=1$ must be considered separately. In this case, we have the following result.

Proposition 7. Let $p$ be a prime. Assume that one of the following conditions holds: 
i) $k<r d^{2}(p-1)$,

ii) $k$ is not a multiple of $p-1$.

Then, $S_{w}^{d}(p) \equiv 0(\bmod p)$.

Proof. If $p=2$ condition ii) cannot hold and if condition i) holds, we can apply the same argument of the proof of the first part of Proposition 6 to get the result.

Now, if $p$ is odd, again each entry in the matrix $S_{w}^{d}(p)$ is a homogeneous polynomial in the $r d^{2}$ variables $a_{i, j}^{l}$. Hence, it is a sum of elements of the form

$$
\sum_{a_{i, j}^{l} \in \mathbb{Z}_{p}} \prod\left(a_{i, j}^{l}\right)^{\beta_{i, j, l}}
$$

We have that $\sum_{i, j, l} \beta_{i, j, l}=k$ so, if $k<r d^{2}(p-1)$ or if it is not a multiple of $p-1$ it follows that some $\beta_{i, j, l}$ is either 0 or not a multiple of $p-1$. In either case the corresponding element is $0(\bmod p)$ due to Lemma 3 and, consequently, each entry in the matrix $S_{w}^{d}(p)$ is also $0(\bmod p)$ as claimed.

Observe that in the previous results we have considered sums of the form

$$
S_{w}^{d}\left(p^{s}\right)=\sum_{A_{1}, \ldots, A_{r} \in M_{p^{s}}^{d}} w\left(A_{1}, \ldots, A_{r}\right)
$$

where all the matrices $A_{i}$ belong to the same matrix ring $M_{p^{s}}^{d}$. The following proposition will be useful in the next section and deals with the case when the matrices $A_{i}$ belong to different matrix rings. First, we introduce some notation. Given a prime $p$, let

$$
S_{w}^{d}\left(p^{s_{1}}, \ldots, p^{s_{r}}\right):=\sum_{A_{i} \in M_{p^{d}}^{d}} w\left(A_{1}, \ldots, A_{r}\right)
$$

If $s_{1}=\cdots=s_{r}=s$, then $S_{w}^{d}\left(p^{s_{1}}, \ldots, p^{s_{r}}\right)=S_{w}^{d}\left(p^{s}\right)$ and we are in the previous situation.

Proposition 8. With the previous notation, if $s_{1}>1$, then

$$
S_{w}^{d}\left(p^{s_{1}+1}, p^{s_{2}}, \ldots, p^{s_{r}}\right) \equiv p^{d^{2}} S_{w}^{d}\left(p^{s_{1}}, p^{s_{2}}, \ldots, p^{s_{r}}\right) \quad\left(\bmod p^{s_{1}+1}\right) .
$$


Proof. Since $s_{1}>1$ we have that $2 s_{1}>s_{1}+1$ so, due to Lemma 1

$$
\begin{aligned}
& S_{w}^{d}\left(p^{s_{1}+1}, p^{s_{2}}, \ldots, p^{s_{t}}\right)=\sum_{A_{1} \in M_{p^{s_{1}+1}}^{d}} w\left(A_{1}, \ldots, A_{t}\right)= \\
& A_{i} \in M_{p^{s_{i}}}^{d_{i}} \\
& =\sum_{\substack{B \in M_{p^{s_{1}}, C \in M_{p}^{d}}^{d_{i}} \\
A_{i} \in M_{p^{s_{i}}}^{d}}} w\left(B+p^{s_{1}} C, A_{2}, \ldots, A_{r}\right) \equiv \\
& \equiv \sum_{\substack{B \in M_{p^{s_{1}}}^{d_{1}}, C \in M_{p}^{d} \\
A_{i} \in M_{p^{s_{i}}}^{d}}}\left(w\left(B, A_{2}, \ldots, A_{r}\right)+p^{s_{1}} \sum_{l} w_{l}\left(B, C, A_{2}, \ldots, A_{r}\right)\right)= \\
& =p^{d^{2}} S_{w}^{d}\left(p^{s_{1}}, \ldots, p^{s_{r}}\right)+p^{s_{1}} \sum_{l} \sum_{\substack{B \in M_{p^{s_{1}}}^{d}, C \in M_{p}^{d} \\
A_{i} \in M_{p^{s_{i}}}^{d}}} w_{l}\left(B, C, A_{2}, \ldots, A_{r}\right) \\
& \left(\bmod p^{s_{1}+1}\right) .
\end{aligned}
$$

Where $w_{l}\left(x, y, x_{2}, \ldots, x_{r}\right)$ denotes the monomial $w\left(x_{1}, x_{2}, \ldots, x_{r}\right)$ where the $l-$ th ocurrence of the term $x_{1}$ is substituted by $y$ and the remaining ones by $x$ (for instance, $w\left(x_{1}, x_{2}\right)=x_{1}^{2} x_{2} x_{1}$ gives us $w_{1}\left(x, y, x_{2}\right)=y x x_{2} x, w_{2}\left(x, y, x_{2}\right)=$ $\left.x y x_{2} x, w_{3}\left(x, y, x_{2}\right)=x^{2} x_{2} y\right)$.

But, for every $l$, the monomial $w_{l}\left(B, C, A_{2}, \ldots, A_{r}\right)$ contains $C$ only once and with exponent 1. Hence,

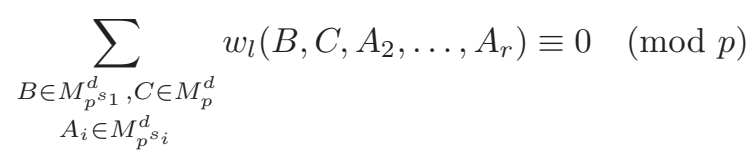

because $S_{1}^{d}(p) \equiv 0(\bmod p)$ and the result follows.

The following corollary in now straightforward.

Corollary 3. Assume that $S_{w}^{d}\left(p^{s}\right) \equiv 0\left(\bmod p^{s}\right)$. Let us consider $s_{1} \geq s_{2} \geq \cdots \geq$ $s_{r}=s$. Then,

$$
S_{w}^{d}\left(p^{s_{1}}, \ldots, p^{s_{r}}\right) \equiv 0 \quad\left(\bmod p^{s_{1}}\right) .
$$

Proof. Just apply the previous proposition repeatedly.

\section{Power sums of matrices over a finite commutative Ring}

In this section we will use the results from Section 3 to compute $S_{k}^{d}(R)$ for an arbitrary finite commutative ring $R$ in many cases.

First of all, note that if $\operatorname{char}(R)=n=p_{1}^{s_{1}} \cdots p_{t}^{s_{t}}$, then $R \cong R_{1} \times \cdots \times R_{t}$, where $\operatorname{char}\left(R_{i}\right)=p_{i}^{s_{i}}$ and each $R_{i}$ is a subring of characteristic $p_{i}^{s_{i}}$ and, in particular, a $Z_{p_{i}^{s_{i}}}-$ module. This allows us to restrict ourselves to the case when $\operatorname{char}(R)$ is a prime power.

The simplest case arises when $R$ is a free $\mathbb{Z}_{p^{s}}$-module for an odd prime $p$.

Proposition 9. Let $p$ be an odd prime and let $R$ be a finite commutative ring of characteristic $p^{s}$, such that $R$ is a free $\mathbb{Z}_{p^{s}}$-module of rank $r$. Then,

i) If $s>1, S_{k}^{d}(R)=0$ for every $k \geq 1$ and $d \geq 2$. 
ii) If $s=1, S_{k}^{d}(R)=0$ for every $d \geq 2$ and $k$ such that either $k<r d^{2}(p-1)$ or $k$ is not a multiple of $p-1$.

Proof. Note that under the previous assumptions and using Proposition 5 or Proposition 7 (depending on whether $s>1$ or $s=1$ ), it follows that

$$
\sum_{A_{1}, \ldots, A_{r} \in M_{p^{s}}^{d}}\left(x_{1} A_{1}+\cdots+x_{r} A_{r}\right)^{k} \equiv 0 \quad\left(\bmod p^{s}\right)
$$

because each entry of such a matrix is a polynomial in $x_{1}, \ldots, x_{r}$ whose coefficients are 0 modulo $p^{s}$.

Consequently, for every $g_{1}, \ldots, g_{r} \in R$ we have that

$$
\sum_{A_{1}, \ldots, A_{r} \in M_{p^{s}}^{d}}\left(g_{1} A_{1}+\cdots+g_{r} A_{r}\right)^{k}=0 .
$$

Now, since $R$ is free of rank $r$ we can take a basis $g_{1}, \ldots, g_{r}$ of $R$ so that $M_{p^{s}}^{d}=$ $\left\{g_{1} A_{1}+\cdots+g_{r} A_{r} \mid A_{i} \in M_{p^{s}}^{d}\right\}$. Therefore

$$
S_{k}^{d}(R)=\sum_{A_{1}, \ldots, A_{r} \in M_{p^{s}}^{d}}\left(g_{1} A_{1}+\cdots+g_{r} A_{r}\right)^{k} .
$$

This concludes the proof.

If $p=2$, we have the following version of Proposition 9

Proposition 10. Let $R$ be a finite commutative ring of characteristic $2^{s}$, such that $R$ is a free $\mathbb{Z}_{2^{s}}$-module of rank $r$. Then,

i) If $s>1, S_{k}^{d}(R)=0$ for every $d \geq 2$ and $k$ such that $k \leq r d^{2}$ or $k>r d^{2}$ with $k+r d^{2}$ even.

ii) If $s=1, S_{k}^{d}(R)=0$ for every $d \geq 2$ and $k$ such that either $k<r d^{2}$.

Proof. The proof is similar to that of Proposition 9 using Proposition 6 or Proposition 7 depending on whether $s>1$ or $s=1$.

Remark. Note that if $R$ is a finite commutative ring of characteristic $p^{s}$ and $s=1$, then $R$ is necessarily free. Consequently, to study the non-free case we may assume that $s>1$.

Assume that elements $g_{1}, \ldots, g_{r}$ form a minimal set of generators of a non-free $\mathbb{Z}_{p^{s}}$-module $R$. Since $R$ is non-free and $\operatorname{char}(R)=p^{s}$, it follows that $r>1$ and also $s>1$. For every $i \in\{1, \ldots, r\}$ let $1 \leq s_{i} \leq s$ be minimal such that $p^{s_{i}} g_{i}=0$. Note that it must be $s_{i}=s$ for some $i$ and $s_{j}<s$ for some $j$. There is no loss of generality in assuming that $s=s_{1} \geq \cdots \geq s_{r}$ and at least one of the inequalities is strict. Note that $p^{s_{1}}, \ldots, p^{s_{r}}$ are the invariant factors of the $\mathbb{Z}$-module $R$. With this notation we have the following result extending Proposition 9

Proposition 11. Let $p$ be an odd prime and let $R$ be a finite commutative ring of characteristic $p^{s}$, such that $R$ is a non-free $\mathbb{Z}_{p^{s}}-$ module. Then,

i) If $s_{r}>1, S_{k}^{d}(R)=0$ for every $k \geq 1$ and $d \geq 2$.

ii) If $s_{r}=1, S_{k}^{d}(R)=0$ for every $d \geq 2$ and $k$ such that either $k<r d^{2}(p-1)$ or $k$ is not a multiple of $p-1$. 
Proof. First of all, observe that

$$
S_{k}^{d}(R)=\sum_{A_{i} \in M_{p^{d}}^{s_{i}}}\left(g_{1} A_{1}+\cdots+g_{r} A_{r}\right)^{k} .
$$

In both situations i) and ii) it follows that $S_{w}^{d}\left(p^{s_{r}}\right) \equiv 0\left(\bmod p^{s_{r}}\right)$. Moreover, we are in the conditions of Corollary 3. so it follows that $S_{w}^{d}\left(p^{s}, p^{s_{2}}, \ldots, p^{s_{r}}\right) \equiv 0$ $\left(\bmod p^{s}\right)$. Consequently all the coefficients of the above sum are 0 modulo $p^{s}$ and the result follows.

The corresponding result for $p=2$ is as follows.

Proposition 12. Let $R$ be a finite commutative ring of characteristic $2^{s}$, such that $R$ is a non-free $\mathbb{Z}_{p^{s}}-$ module. Then,

i) If $s_{r}>1, S_{k}^{d}(R)=0$ for every $d \geq 2$ and $k$ such that $k \leq r d^{2}$ or $k>r d^{2}$ with $k+r d^{2}$ even.

ii) If $s_{r}=1, S_{k}^{d}(R)=0$ for every $d \geq 2$ and $k$ such that either $k<r d^{2}$.

Proof. It is identical to the proof of Proposition 11

\section{Conjectures and Further WOrK}

Given a finite commutative ring $R$ of characteristic $n$, we have seen in the last section that $S_{k}^{d}(R)=0$ for many values of $k, d$ and $n$. In this section we present two conjectures based on strong computational evidence which, being true, would let us to give a general result about $S_{k}^{d}(R)$.

With the notation from the previous section, given an $r$-tuple of integers $\kappa=$ $\left(k_{1}, \ldots, k_{r}\right)$, we consider the set of monomials in the non-commuting variables $\left\{x_{1}, \ldots, x_{r}\right\}$

$$
\Omega_{\kappa}:=\left\{w: \operatorname{deg}_{x_{i}}(w)=k_{i}, \text { for every } i\right\} .
$$

The following conjectures are based on computational evidence.

Conjecture 2. With the previous notation, let $s_{1} \geq s_{2} \geq \cdots \geq s_{r}$. Then

$$
S_{w}^{d}\left(p^{s_{1}}, p^{s_{2}}, \ldots, p^{s_{r}}\right) \equiv 0 \quad\left(\bmod p^{s_{1}}\right),
$$

unless $d=p=2$ and $s_{i}=1$ for all $i$.

Conjecture 3. If $p=2=d$ and $r>1$ then for every $\kappa \in \mathbb{N}^{r}$

$$
\sum_{w \in \Omega_{\kappa}} \sum_{A_{i} \in M_{2}^{d}} w\left(A_{1}, \ldots, A_{r}\right) \equiv 0 \quad(\bmod 2) .
$$

The next lemma extends Lemma 2 in some sense. Its proof is straightforward.

Lemma 5. Let $R_{1}$ and $R_{2}$ be finite commutative rings, and let $R=R_{1} \times R_{2}$ be its direct product. Then

$$
S_{k}^{d}(R)=\left(\operatorname{card}\left(R_{2}\right)^{d^{2}} \cdot S_{k}^{d}\left(R_{1}\right), \operatorname{card}\left(R_{1}\right)^{d^{2}} \cdot S_{k}^{d}\left(R_{2}\right)\right) \in \mathbb{M}_{d}\left(R_{1}\right) \times \mathbb{M}_{d}\left(R_{2}\right)
$$

Now, the following proposition would follow from Conjectures 2 and 3 .

Proposition 13. Let $R$ be a finite commutative ring of characteristisc $p^{s}$ for some prime $p$. Then $S_{k}^{d}(R)=0$ unless $d=2, R=\mathbb{Z} / 2 \mathbb{Z}$ and $1<k \equiv-1,0,1(\bmod 6)$. Moreover, in this case $S_{k}^{d}(R)=I_{2}$. 
Proof. Assume that $\left\langle g_{1} \ldots, g_{r}\right\rangle$ is a minimal set of generators of $R$ as $\mathbb{Z}_{p^{s}}$-module. Let $s=s_{1} \geq s_{2} \geq \cdots \geq s_{r}$ be integers such that the order of $g_{i}$ is $p^{s_{i}}$; i.e., $s_{1}, \ldots, s_{r}$ are minimal such that $p^{s_{i}} g_{i}=0$.

In this situation,

$$
S_{k}^{d}(R)=\sum_{A_{i} \in M_{p^{s_{i}}}^{d}}\left(g_{1} A_{1}+\ldots+g_{r} A_{r}\right)^{k}=0,
$$

unless $d=p=2, s=r=1$ and $1<k \equiv-1,0,1(\bmod 6)$ due to Conjecture 2,

On the other hand, if $d=p=2, s=r=1$ and $1<k \equiv-1,0,1(\bmod 6)$ it follows that

$$
S_{k}^{2}(R)=\sum_{A \in M_{2}^{2}}\left(g_{1} A\right)^{k}=\left(\begin{array}{cc}
g_{1}^{k} & 0 \\
0 & g_{1}^{k}
\end{array}\right) .
$$

But since in this case $R=\left\{0, g_{1}\right\}$, there are only two possibilities: $g_{1}^{2}=g_{1}$ (and hence $R=\mathbb{Z} / 2 \mathbb{Z}$ ) or $g_{1}^{2}=0$ and the result follows.

Finally, the next general result holds provided Conjectures 2 and 3 are correct. It is Conjecture 1 as stated in the introduction to the paper.

Theorem 4. Let $d>1$ and let $R$ be a finite commutative ring. Then $S_{k}^{d}(R)=0$ unless the following conditions hold:

(1) $d=2$,

(2) $\operatorname{card}(R) \equiv 2(\bmod 4)$ and $1<k \equiv-1,0,1(\bmod 6)$,

(3) The unique element $e \in R \backslash\{0\}$ such that $2 e=0$ is idempotent.

Moreover, in this case

$$
S_{k}^{d}(R)=\left(\begin{array}{ll}
e & 0 \\
0 & e
\end{array}\right)
$$

Proof. First, observe that if $\operatorname{card}(R) \equiv 2(\bmod 4)$, then $R$ has $2 m$ elements, where $m$ is odd. Therefore, the 2 -primary component of the additive group $R$ has only two elements, and so there is a unique element $e \in R$ of additive order 2 .

Now, if $R$ is of characteristic $p^{s}$ for some prime, the result follows from the above proposition. Hence, we assume that $R$ has composite characteristic. Let $R=R_{1} \times R_{2}$ with $R_{1}$ the zero ring or $\operatorname{char}\left(R_{1}\right)=2^{s}$ and $\operatorname{char}\left(R_{2}\right)$ odd. Due to Lemma 5 and Proposition 13 it follows that $S_{k}^{d}(R)=\left(\operatorname{card}\left(R_{2}\right)^{d^{2}} \cdot S_{k}^{d}\left(R_{1}\right), 0\right)$.

Now, $S_{k}^{d}\left(R_{1}\right)=0$ unless $d=2=p, R_{1}=\mathbb{Z} / 2 \mathbb{Z}$ and $1<k \equiv-1,0,1(\bmod 6)$ in which case

$$
S_{k}^{d}(R)=\left(\left(\begin{array}{ll}
1 & 0 \\
0 & 1
\end{array}\right),\left(\begin{array}{ll}
0 & 0 \\
0 & 0
\end{array}\right)\right)=\left(\begin{array}{ll}
e & 0 \\
0 & e
\end{array}\right),
$$

where $e=(1,0) \in R_{1} \times R_{2}$ is the only idempotent of $R$ such that $2 e=0$.

Remark. Note that if, in addition, $R$ is unital then the element $e$ from the previous theorem is just $e=\frac{\operatorname{card}(R)}{2} \cdot 1_{R}$. Also note that if $S_{k}^{d}(R) \neq 0$, then $R \cong \mathbb{Z} / 2 \mathbb{Z} \times R_{2}$ with $\operatorname{card}\left(R_{2}\right)$ odd or $R_{2}=\{0\}$.

We close the paper with a final conjecture.

Conjecture 4. Theorem 4 remains true if $R$ is non-commutative. 


\section{REFERENCES}

[1] J.V. Brawley, L. Carlitz, J. Levine. Power sums of matrices over a finite field. Duke Math. J., 41:9-24, 1974.

[2] L. Carlitz. The Staudt-Clausen theorem. Math. Mag., 34:131-146, 1960-1961.

[3] P. Fortuny, J.M. Grau, A. M. Oller-Marcén. A von Staudt-type result for $\sum_{z \in \mathbb{Z}_{n}[i]} z^{k}$. Monatsh. Math. DOI: 10.1007/s00605-015-0736-5, 2015.

[4] J.M. Grau, C. Miguel, A.M. Oller-Marcén. On the structure of quaternion rings over $\mathbb{Z} / n \mathbb{Z}$. Adv. Appl. Clifford Algebr., DOI: 10.1007/s00006-015-0544-y, 2015.

[5] J.M. Grau, P. Moree, A.M. Oller-Marcén. Solutions of the congruence $\sum_{k=1}^{n} k^{f(n)} \equiv 0$ $(\bmod n)$. Math. Nachr., to appear.

[6] J.M. Grau, A.M. Oller-Marcén, J. Sondow. On the congruence $1^{m}+2^{m}+\cdots+m^{m} \equiv n$ $(\bmod m)$ with $n \mid m$ Monatsh. Math., DOI 10.1007/s00605-014-0660-0, 2014.

[7] P. Moree. On a theorem of Carlitz-von Staudt. C. R. Math. Rep. Acad. Sci. Canada, 16(4):166-170, 1994.

[8] N.J.A. Sloane. The On-Line Encyclopedia of Integer Sequences. https://oeis.org.

[9] K.G.C. von Staudt. Beweis eines Lehrsatzes die Bernoullischen Zahlen betreffend. J. Reine Angew. Math, 21:372-374, 1840.

Departamento de Matemáticas, Universidad de Oviedo, Avda. Calvo Sotelo, s/n, 33007 Oviedo, Spain

E-mail address: fortunypedro@uniovi.es

Departamento de Matemáticas, Universidad de Oviedo, Avda. Calvo Sotelo, s/n, 33007 Oviedo, Spain

E-mail address: grau@uniovi.es

Centro Universitario de la Defensa de zaragoza, Ctra. Huesca s/n, 50090 Zaragoza, SPAIN

E-mail address: oller@unizar.es

Departamento de Matemáticas, Universidad de Oviedo, Avda. Calvo Sotelo, s/n, 33007 Oviedo, Spain

E-mail address: rua@uniovi.es 